\title{
Shear thinning and shear thickening of a confined suspension of vesicles
}

\author{
A. Nait Ouhra,,${ }^{1,2, *}$ A. Farutin, ${ }^{1}$ O. Aouane, ${ }^{1,3}$ H. Ez-Zahraouy, ${ }^{2}$ A. Benyoussef,${ }^{2,4}$ and C. Misbah ${ }^{1, \dagger}$ \\ ${ }^{1}$ Laboratoire Interdisciplinaire de Physique, Université Grenoble Alpes, CNRS, LIPhy, 38000 Grenoble, France \\ ${ }^{2}$ Laboratoire de Matière Condensée et Sciences Interdisciplinaires, Faculty of Sciences, Mohammed V University of Rabat, 1014 Morocco \\ ${ }^{3}$ Forschungszentrum Jülich GmbH, Helmholtz-Institute Erlangen-Nürnberg for Renewable Energy (IEK-11), \\ Dynamics of Complex Fluids and Interfaces, Fürther Straße 248, 90429 Nürnberg, Germany \\ ${ }^{4}$ Hassan II Academy of Science and Technology, Rabat, 10220 Morocco
}

(Received 1 August 2017; revised manuscript received 28 November 2017; published 12 January 2018)

\begin{abstract}
Widely regarded as an interesting model system for studying flow properties of blood, vesicles are closed membranes of phospholipids that mimic the cytoplasmic membranes of red blood cells. In this study we analyze the rheology of a suspension of vesicles in a confined geometry: the suspension, bound by two planar rigid walls on each side, is subject to a shear flow. Flow properties are then analyzed as a function of shear rate $\dot{\gamma}$, the concentration of the suspension $\phi$, and the viscosity contrast $\lambda=\eta_{\text {in }} / \eta_{\text {out }}$, where $\eta_{\text {in }}$ and $\eta_{\text {out }}$ are the fluid viscosities of the inner and outer fluids, respectively. We find that the apparent (or effective viscosity) of the suspension exhibits both shear thinning (decreasing viscosity with shear rate) or shear thickening (increasing viscosity with shear rate) in the same concentration range. The shear thinning or thickening behaviors appear as subtle phenomena, dependant on viscosity contrast $\lambda$. We provide physical arguments on the origins of these behaviors.
\end{abstract}

DOI: 10.1103/PhysRevE.97.012404

\section{INTRODUCTION}

Vesicles are closed fluid membranes suspended in an aqueous medium. They are made of bilayers of phospholipids, each having a hydrophilic head and two hydrophobic tails [1]. Vesicles represent an attractive biomimetic system for studying the viscoelastic properties of real cells, such as red blood cells (RBCs). Blood rheology for unconfined flow has been of considerable interest both for experimental and for theoretical studies. Experimentally, blood shows a shear thinning behavior associated with the dissociation of RBC rouleaux upon increasing shear rate [2-7]. Theoretically, several phenomenological continuum viscoelastic models (for a review see [8]) have been used to characterize the rheology of blood flow. However, these models are based on assumptions that are difficult to confirm or justify. Up until now, the general strategy has consisted of adding progressively new ingredients, such as generalized models with shear-rate dependent viscosity, yield-stress behaviors, elastic stress, etc., in order to account for diverse properties of blood flow, which depend on regimes of flow strength.

As for any complex fluid [9], the rheology of blood escapes the traditional laws for simple fluids. Its complex character stems from an intimate link between the microscales (carried by RBC shapes and dynamics) and macroscales. In principle, a firm understanding of rheology should emerge by explicitly taking RBC shapes and dynamics into consideration. The increase in computational power combined with the development of many numerical techniques has opened the way towards

\footnotetext{
*abdessamad.nait-ouhra@univ-grenoble-alpes.fr

†chaouqi.misbah@univ-grenoble-alpes.fr
}

numerical studies of blood flow properties by allowing us to account for several hundreds or thousands of cells at a time. Many studies have been devoted to the rheology of dilute suspensions of vesicles or capsules (another model of elastic shells mimicking RBCs), theoretically [10-12], numerically [13-19], and experimentally [20,21]. More recently, several simulations have been devoted to the study of more concentrated suspensions [22-26].

A less-treated issue concerns rheology in a confined geometry. Recently, the subject was approached [27] by submitting a confined suspension to a shear flow. It was reported that the suspension exhibits several ordered structures with an important impact on rheology. For example the normalized effective viscosity, defined as $[\eta]=\frac{\eta-\eta_{\text {out }}}{\eta_{\text {out }} \phi}$ (with $\eta$ the effective viscosity of the suspension, $\eta_{\text {out }}$ the viscosity of the ambient fluid, and $\phi$ the vesicle concentration) exhibits ample oscillations with respect to $\phi$ and coinciding with the appearance of several ordered structures.

The aforementioned study [27] focused on the evolution of rheological properties as a function of concentration $\phi$ and confinement. The viscosity contrast as well as the strength of the flow were kept fixed. Other studies [28,29] focused on the effect of viscosity contrast $\lambda$ on the dynamics and rheology of vesicle suspensions, while the effect of confinement has been investigated in Ref. [30] in the absence of a viscosity contrast. The evolution of rheology with the flow strength is a classical study that allows us to analyze the shear thinning or shear thickening behavior. In the present systematic study we look at the rheological properties as a function of shear rate $\dot{\gamma}$ by varying the viscosity contrast $\lambda=\eta_{\text {in }} / \eta_{\text {out }}$, where $\eta_{\text {in }}$ and $\eta_{\text {out }}$ are the viscosities of the fluid medium inside and outside the vesicle, respectively. An interesting feature is discovered: the suspension can exhibit both shear thinning and shear 
thickening depending on $\lambda$. Unlike the classical shear thinning effect for blood, which is mostly explained by the shearinduced dissociation of $\mathrm{RBC}$ rouleaux (the rest being attributed to the effects of cell deformability), here, no rouleaux are allowed to form due to the absence of any nonhydrodynamic interactions between cells. Instead, the shear thinning behavior comes exclusively from the way the suspension organizes itself as a function of the shear rate. It is sufficient to modify the viscosity of the enclosed fluid of the vesicles for the rheological behavior of the suspension to switch from shear thinning to shear thickening.

We shall consider a 2D suspension of vesicles. A preliminary study presented in [27] has shown that the same type of order of the suspended entities takes place also in 3D with the same qualitative behavior regarding the changes of the effective viscosity with the concentration. This is why we believe that the overall behavior presented here should survive in $3 \mathrm{D}$. The efficiency of 2D simulations from the computational time point of view allows one to explore several ranges of parameters in order to identify the relevant regimes of interest.

The article is organized as follows. The model and simulation method are presented in Sec. II. Section III contains the main results and their discussion. We discuss the shearthinning and shear thickening behaviors and their origins, as well as the transition from shear thinning to shear thickening. Section IV is devoted to the conclusion and perspectives.

\section{MODEL AND SIMULATION METHOD}

\section{A. Membrane model}

We consider a set of $2 \mathrm{D}$ vesicles inside a channel, bounded by two straight rigid walls located at $y=-W / 2$ and $y=W / 2$, where $W$ is the channel width, under plane shear flow $v_{x}^{0}=\dot{\gamma} y$ with $v_{x}^{0}$ the component along the flow direction of the imposed flow $\mathbf{v}^{0}$. Periodic boundary conditions are used along $x$, and the box size in this direction is selected as the same manner as in [19]. The membrane of vesicles acts on the fluid via bending force and tension force, and the total one is given by

$$
\mathbf{f}_{\mathrm{mem} \rightarrow \mathrm{flu}}=\kappa\left(\frac{d^{2} c}{d s^{2}}+\frac{1}{2} c^{3}\right) \mathbf{n}-\zeta c \mathbf{n}+\frac{d \zeta}{d s} \mathbf{t} .
$$

This force (Eq. (1) is derived in 2D in [31]) is obtained from the functional derivative of the Helfrich [32] bending energy $E=\frac{\kappa}{2} \int_{\text {mem }} c^{2} d s+\int_{\text {mem }} \zeta d s$, which includes a tension-like term $\zeta$ in order to fulfill the local arclength conservation constraint. Strictly speaking $\zeta$ is a Lagrange multiplier; $c$ is the membrane curvature, $s$ is the curvilinear coordinate along the membrane, $\mathbf{n}$ and $\mathbf{t}$ are the unit normal and tangent vectors, and $\kappa$ is the membrane bending rigidity (typical value of vesicles is of about a fraction of an $\mathrm{eV}$ ). The enclosed area $A$ is conserved automatically thanks to the fluid incompressibility (it is enforced by the pressure term in the flow equations written below).

\section{B. Boundary integral formulation}

Vesicles and RBCs are small enough and their speeds (say in vivo for RBCs) are also small enough that the corresponding Reynolds number is small (typically in the range $10^{-4}$ to $10^{-2}$ ).
We can safely consider the limit of a vanishing Reynolds number (the Stokes limit), where the flow is governed by viscous forces (inertia is neglected). The fluid inside and outside the cells is thus described by the Stokes equations:

$$
\begin{aligned}
-\nabla p+\eta_{i} \nabla^{2} \mathbf{v} & =0, \\
\nabla \cdot \mathbf{v} & =0,
\end{aligned}
$$

where $p$ is the pressure and $\mathbf{v}$ is the velocity field. $\eta_{i}=\eta_{\text {in }}$ $\left(\eta_{\text {out }}\right)$ for the inner (outer) fluid. The continuity of the velocity field and the stress balance are to be satisfied at the membrane. The surface area (perimeter in 2D) of the membrane is locally conserved. Due to the linearity of the Stokes equations, we can use the boundary integral method [33] to formulate the dynamics of the vesicles. This method is based on the use of the Green's function technique. We use here the Green's function which fulfills the no-slip boundary condition at the bounding walls [19]. More precisely, for a point $\mathbf{r}_{0}$ which belongs to a membrane, the velocity $\mathbf{v}\left(\mathbf{r}_{0}\right)$ of this point has the following dimensionless expression:

$$
\begin{aligned}
\mathbf{v}\left(\mathbf{r}_{0}\right)= & \frac{2}{1+\lambda} \mathbf{v}^{0}\left(\mathbf{r}_{0}\right) \\
& +\frac{1}{2 \pi C_{\kappa}(1+\lambda)} \int_{\mathrm{mem}} d s \mathbf{f}_{\mathrm{mem} \rightarrow \mathrm{flu}}(\mathbf{r}) \cdot \mathrm{G}^{2 w}\left(\mathbf{r}, \mathbf{r}_{0}\right) \\
& +\frac{1-\lambda}{2 \pi(1+\lambda)} \int_{\mathrm{mem}} d s \mathbf{v}(\mathbf{r}) \cdot \mathrm{T}^{2 w}\left(\mathbf{r}, \mathbf{r}_{0}\right) \cdot \mathbf{n}(\mathbf{r}),
\end{aligned}
$$

where we have used the following scales:

(1) $R_{0}=\sqrt{A / \pi}$ for the length.

(2) $U=\dot{\gamma} R_{0}$ for the velocity.

(3) $\kappa / R_{0}^{3}$ for the force.

The dimensionless parameters $C_{\kappa}$ and $\lambda$ entering Eq. (3) are discussed below and the dimensionless force $\mathbf{f}_{\mathrm{mem} \rightarrow \text { flu }}$ is given by the following expression (where we keep the same notations for simplicity):

$$
\mathbf{f}_{\mathrm{mem} \rightarrow \mathrm{flu}}=\left(\frac{d^{2} c}{d s^{2}}+\frac{1}{2} c^{3}\right) \mathbf{n}-\zeta c \mathbf{n}+\frac{d \zeta}{d s} \mathbf{t} .
$$

The still unknown Lagrange multiplier $\zeta$ is obtained by imposing a divergence-free velocity along the membrane, expressing the membrane incompressibility. The numerical method of imposing membrane incompressibility follows closely that presented in [14].

The displacement in the course of time of the vesicle membranes is obtained by updating the discretization points after each time iteration, using an Euler scheme, $\mathbf{r}_{0}(t+d t)=$ $\mathbf{v}(t) d t+\mathbf{r}_{0}(t)$

\section{Dimensionless parameters}

The full problem is described by several dimensionless numbers, which are as follows:

(1) The capillary number $C_{\kappa}$ measuring the flow strength over the bending energy of the membrane. In other words, $C_{\kappa}$ controls how the shapes of vesicles deform in response to an applied external flow:

$$
C_{\kappa}=\frac{\eta_{\mathrm{out}} \dot{\gamma} R_{0}^{3}}{\kappa} \equiv \dot{\gamma} \tau_{c},
$$


where $\tau_{c}$ is the typical time needed for the vesicle to recover its equilibrium shape after cessation of flow. Time will be measured hereafter in units of $\tau_{c}$. In this paper the capillary number is varied in the wide range $C_{\kappa}=1-200$.

(2) The viscosity contrast

$$
\lambda=\frac{\eta_{\text {in }}}{\eta_{\text {out }}} .
$$

We have explored several values of $\lambda$ in the range $\lambda=$ $1-10$; in all of these cases, the suspended entities exhibit tank-treading (TT) motion [19].

These two dimensionless numbers enter Eq. (3) directly and have to be supplemented by three other numbers:

(1) The reduced area

$$
v=(A / \pi) /(L / 2 \pi)^{2},
$$

where $L$ is the vesicle perimeter and $A$ the enclosed area. In most of our simulations we set $v=0.7$ (close enough to that provided in the literature for human 3D RBCs, having a reduced volume of about 0.6 ). However, other values of $v$ have been explored (see below), showing that the main qualitative results are insensitive to this choice.

(2) The degree of confinement

$$
C_{n}=\frac{2 R_{0}}{W}
$$

In this study this parameter is fixed to 0.4 corresponding to $W=5 R_{0}$. In the regime of a single file studied in [27] we did not notice any major difference between different confinements regarding rheology. In addition, the $3 \mathrm{D}$ simulation showed the same trend.

(3) The concentration $\phi$, which is expressed in terms of the area fraction of vesicles (the 2D analog of the volume fraction). In this work, we consider three concentrations scanning a range from the dilute regime up to what we classify as the semidilute regime. In the dilute regime, we have a singlefile organization, while in the semidilute regime we have a double-file organization. Despite the transition from a singleto double-file solution, the behavior of rheology as a function of shear rate continues to show the same trend, as discussed below.

\section{RESULTS AND DISCUSSION}

\section{A. Shear thinning}

We first analyze the effect of the capillary number $C_{\kappa}$ on the rheology of a vesicle suspension with a viscosity contrast $\lambda=$ 1. The first rheological quantity of interest is the normalized effective viscosity $[\eta][14,19]$ :

$$
\begin{aligned}
{[\eta] \equiv } & \frac{\eta-\eta_{\text {out }}}{\eta_{\text {out }} \phi} \\
= & \frac{1}{\eta_{\text {out }} A \dot{\gamma}}\left[\int_{\text {mem }} d s y f_{\text {flu } \rightarrow \text { mem }, x}\right. \\
& \left.+\eta_{\text {out }}(\lambda-1) \int_{\text {mem }} d s\left(n_{x} v_{y}+n_{y} v_{x}\right)\right] .
\end{aligned}
$$

This expression is the extension to vesicles of the Batchelor [34] result originally derived for suspensions of rigid particles. The second quantity of interest is the normalized normal stress (a)

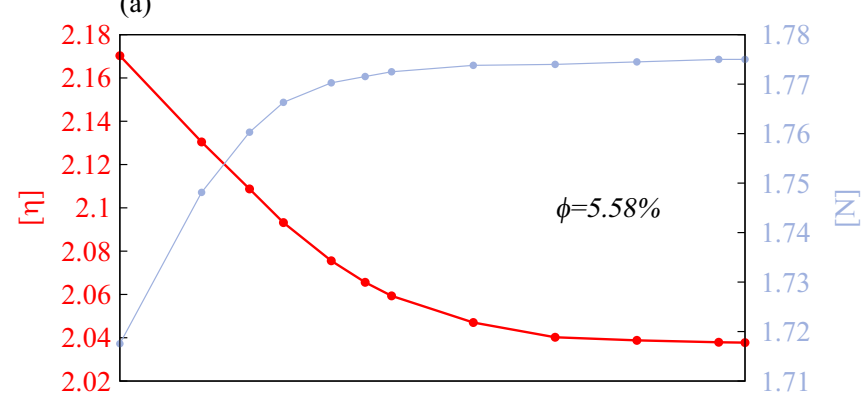

(b)

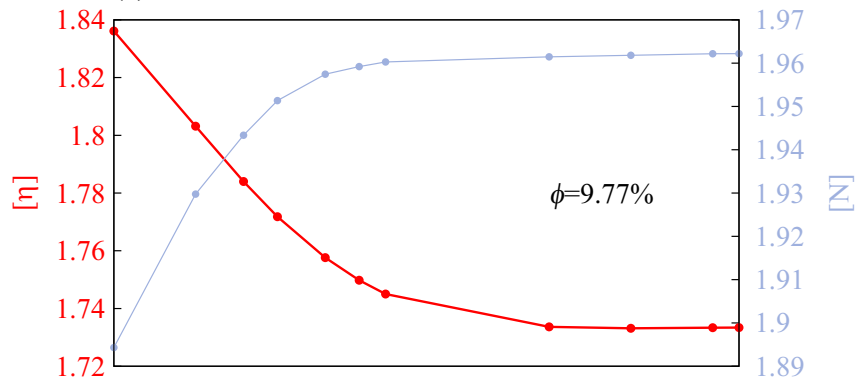

(c)

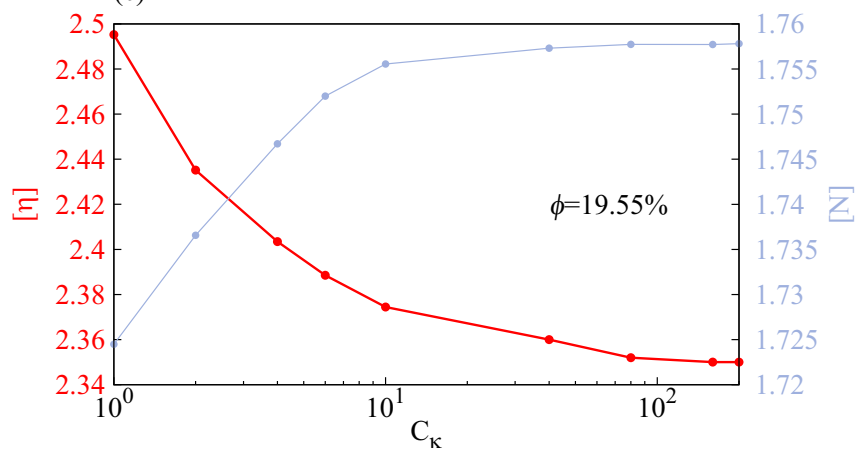

FIG. 1. The normalized effective viscosity $[\eta]$ and the normalized normal stress difference $[N]$ as a function of capillary number $C_{\kappa}$ for different concentrations. For all explored concentrations, the suspension exhibits a shear thinning behavior.

difference:

$$
[N] \equiv \frac{\left\langle\sigma_{x x}\right\rangle-\left\langle\sigma_{y y}\right\rangle}{\left(\eta-\eta_{\text {out }}\right) \dot{\gamma}} .
$$

When $\lambda=1$ (considered in this section), the vesicles exhibit a TT motion (the membrane circulates around the interior of the vesicle). In this case the systematic study of the suspension under different capillary numbers reveals a shear thinning behavior. The results are shown in Fig. 1, for the concentrations $\phi=5.58 \%, \phi=9.77 \%$, and $\phi=19.55 \%$. It is interesting to note that blood also exhibits a shear thinning behavior, which is mostly related to dissociation of $\mathrm{RBC}$ rouleaux, but here rouleaux are absent from the beginning because we do not include nonhydrodynamic interactions between vesicles in our model. The second source of the shear thinning behavior of blood was attributed to RBC deformation [7,14,35,36]. In the present study, we show that the changes of the orientation angle of the vesicles can also affect the effective viscosity of the suspension. We will see below that the situation is quite subtle since a change of the internal viscosity (without changing the overall nature of the vesicle motion or their spatial 


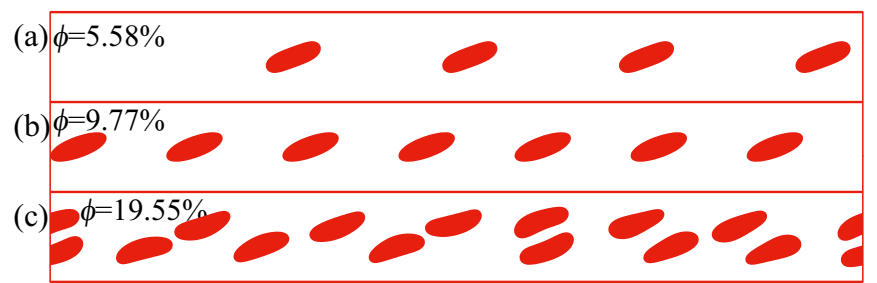

FIG. 2. Typical configurations of a suspension of vesicles for several concentration $\phi$ and typical capillary numbers $C_{\kappa}$. (a) $C_{\kappa}=3$. (b) $C_{\kappa}=80$. (c) $C_{\kappa}=10$.

organization) will lead to shear thickening. It is interesting to observe that the overall trend of the shear thinning/shear thickening behavior is independent of the concentration and of the spatial organization.

Figure 2 shows the spatial organization. Each vesicle is pushed by the walls (lateral migration) towards the centerline. Each vesicle at the center interacts hydrodynamically with its neighbors. The analysis of the flow field around the vesicles gives a precise hint towards the organization [37]. When the concentration exceeds a certain value (typically $\phi \sim 15 \%$; see [27]) addition of new vesicles within the single file becomes unfavorable. The single-file arrangement becomes unstable against the formation of a two-file structure.

Note in Fig. 1 that the normalized effective viscosity (for a given $C_{\kappa}$ ) is not monotonic with concentration. It is higher for $\phi=5.58 \%$ than for $\phi=9.77 \%$ and it is smaller for $\phi=$ $9.77 \%$ than for for $\phi=19.55 \%$. This behavior was initially reported in [27] and some heuristic explanations put forward.

Let us provide now some support to the shear thinning behavior. The first analysis is based on the streamline structure (Fig. 3). Those lines are plotted using stream function $\psi(x, y)$, which is introduced by the definitions

$$
\begin{gathered}
v_{x}=\frac{\partial \psi}{\partial y}, \\
v_{y}=-\frac{\partial \psi}{\partial x} .
\end{gathered}
$$

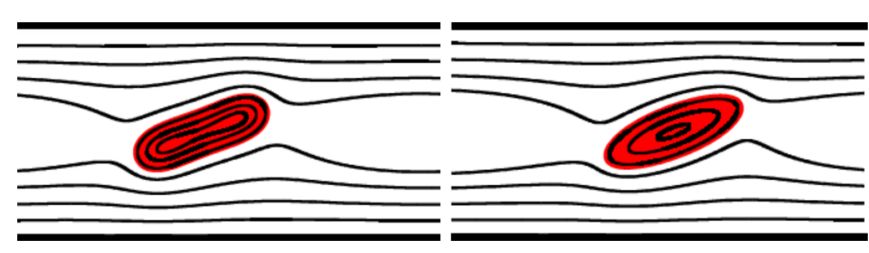

FIG. 3. Streamlines inside and outside a single vesicle of the suspension for the viscosity contrast $\lambda=1$, for two capillary numbers: $C_{\kappa}=1$ (left) and $C_{\kappa}=200$ (right). In this case the inclination angle of the vesicles is almost constant with $C_{\kappa}$, whereas the shape undergoes some moderate change. Owing to this, the streamlines also deform following the deformation of vesicles. We can see in this case that the streamlines inside and outside of the vesicles are more deformed for lower $C_{\kappa}$ than for higher $C_{\kappa}$, in agreement with the shear thinning behavior. The same set of values of the stream function is used for streamlines in left and right panels.

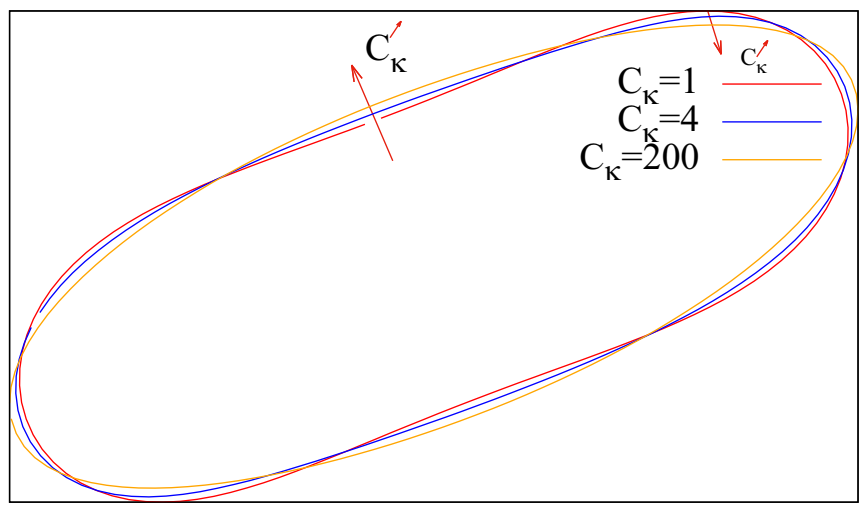

FIG. 4. Steady contours of a vesicle as a function of the capillary number $C_{\kappa}$ for a viscosity contrast $\lambda=1$. The capillary number $C_{\kappa}$ increases in the direction of the arrows.

The stream function is constant along the lower wall, according to (12). Consequently, we can write

$$
\psi\left(x_{0}, y_{0}\right)=\int_{-W / 2}^{y_{0}} v_{x}\left(x_{0}, y\right) d y+C,
$$

where $C$ is an arbitrary constant independent of $x_{0}$ and $y_{0}$. A streamline is defined by equation

$$
\psi\left(x_{0}, y_{0}\right)=K
$$

where $K$ is a constant characterizing the streamline. Knowing $v_{x}$ and $v_{y}$ from the boundary integral equation (as described for example in [38]), we obtain $\psi$ by calculating the integral (13) numerically. Taking a specific desired initial position $\left(x_{0}, y_{0}\right)$ fixes a given value of $K$. The streamlines are obtained by linking the points which have the same given value of stream function $K$.

In Fig. 3, the streamlines are more deformed (and this implies higher dissipation) for small capillary number (left panel) than for the higher one (right panel). In the purpose of providing an argument about the possible source of the shear thinning behavior of these confined suspensions, we can link this phenomenon to the deformation of vesicles. The shape evolution of a vesicle as a function of $C_{\kappa}$ is shown in Fig. 4. The key parameter that controls the deformation of a vesicle by varying the capillary number $C_{\kappa}$ is the Taylor deformation index $D$ defined as

$$
D=\frac{a-b}{a+b},
$$

where $a$ and $b$ are the lengths of the major and minor axes of the vesicle, respectively. It is interesting to note that this parameter decreases with $C_{\kappa}$ (Fig. 5), in marked contrast with the behavior of drops $[39,40]$. At equilibrium the vesicle has a biconcave shape. The straining part of the shear flow tends to align the vesicle along some angle with respect to the flow direction, and this leads to an increase of the long axis of the vesicle. At the same time the short axis expands in order to preserve the constant perimeter by suppressing the dip of the biconcave shape. The expansion of the short axis is larger than that of the long axis, which reduces the Taylor deformation index of the vesicle. The straightening of the concavities and the decrease of the Taylor deformation index means that flow 


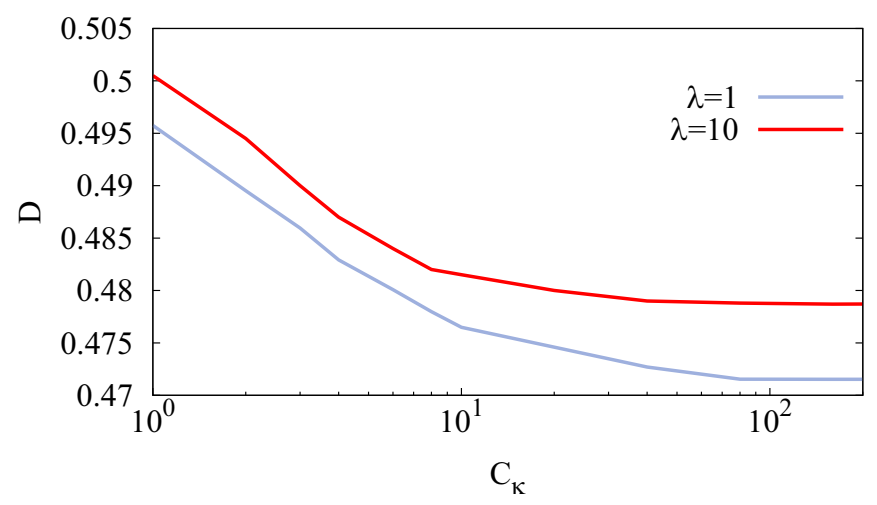

FIG. 5. The Taylor deformation index $D$ as a function of capillary number $C_{\kappa}$ for $\lambda=1$ and $\lambda=10(\phi=5.58 \%)$.

lines inside the vesicle become closer to a circle (Fig. 3), which reduces the dissipation in the internal fluid. This provides a simple explanation to the shear thinning behavior.

\section{B. Shear thickening}

An interesting fact is that the same suspension can exhibit a shear thickening for a different internal viscosity, albeit neither the spatial configuration nor the dynamics of each vesicle is different from the previous case (i.e., each vesicle undergoes a TT motion and the vesicles organize in a regular file).

As we can see in Fig. 6, the suspension becomes more and more viscous with the capillary number $C_{\kappa}$. The spatial organization of the vesicles in this case is shown in Fig. 7. The shear thickening behavior is present despite the fact that the Taylor deformation index $D$ also decreases with $C_{\kappa}$ (see
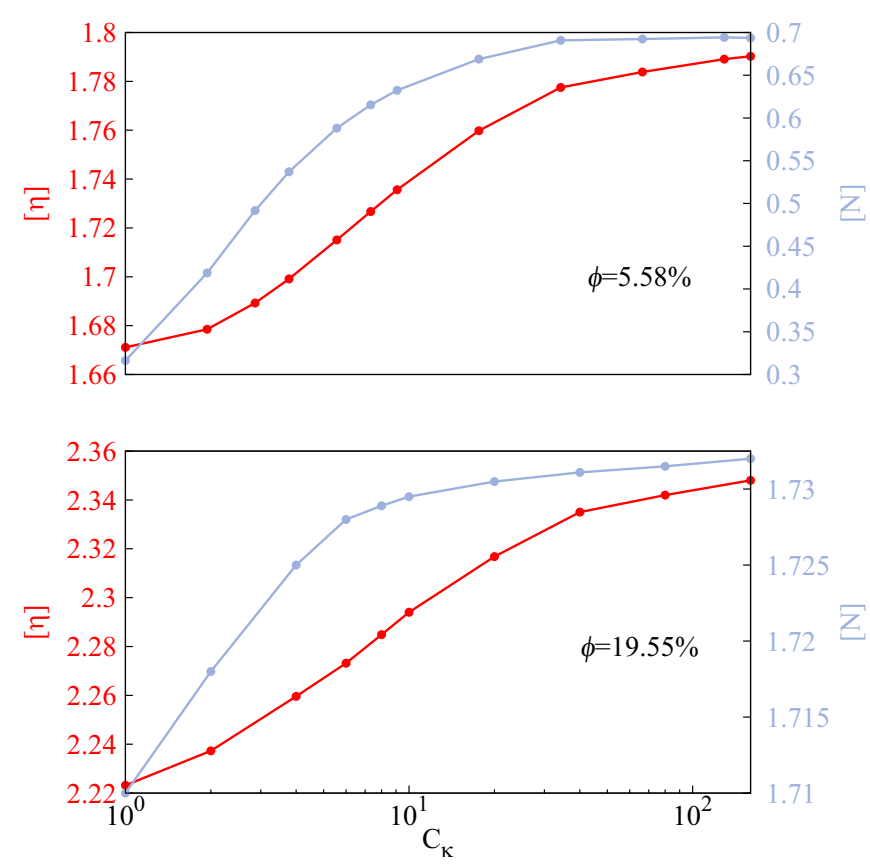

FIG. 6. The normalized effective viscosity $[\eta]$ and the normalized normal stress difference $[N]$ as a function of capillary number $C_{\kappa}$ for $\phi=5.58 \%$ and $\phi=19.55 \%$. For all explored concentrations, the suspension exhibits a shear thickening behavior.

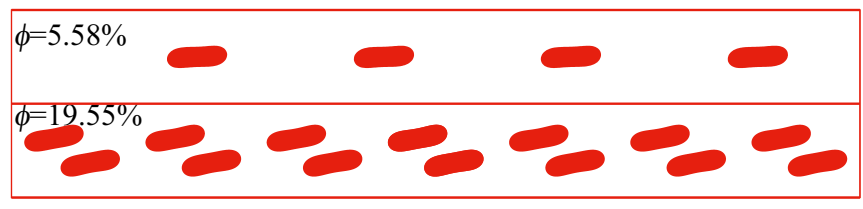

FIG. 7. Typical configurations of a vesicle suspension. Top: $\phi=$ 5.58\%, $C_{\kappa}=1$, and $\lambda=10$. Bottom: $\phi=19.55 \%, C_{\kappa}=1$, and $\lambda=10$.

Fig. 5, red curve). This means that the behavior of the Taylor deformation index is not the only source of the evolution of the viscosity. We have thus attempted to dig further into the reason for this opposite behavior. We found that in this case there are two antagonist effects: (i) the decrease of the Taylor deformation index $D$ with shear rate which leads to shear thinning (as seen before), and (ii) a significant increase of the TT angle with shear rate that causes the vesicles to oppose more resistance to the flow, leading to shear thickening. It turns out that this last effect dominates, leading globally to an increase of the viscosity with the shear rate.

The value $\lambda=10$ is above the critical value for the transition from TT to tumbling (TB) in the unconfined regime [14]. In the presence of confining walls the transition towards TB occurs at higher values of viscosity contrast $\lambda$, as shown in [41]. It is found here that the shear rate also delays the transition towards $\mathrm{TB}$, in that the shear rate tends to significantly increase the TT angle, as seen below.

In order to investigate the reasons causing the shear thickening behavior, we have analyzed the behavior of the the inclination angle of vesicles $\Psi$ as a function of shear rate, expressed by $C_{\kappa}$ in dimensionless units, Fig. 8. Our results show that the angle $\Psi$ increases with $C_{\kappa}$, meaning that the vesicles behave, upon increasing $C_{K}$, more and more as obstacles against the flow, leading to a higher flow resistance.

It turns out that the increase of the inclination angle with capillary number is much more pronounced for high viscosity contrast than for low ones. This is a consequence of the fact that for small $\lambda$ the inclination angle is quite close to its maximum possible value, namely $\pi / 4$, which corresponds to the elongational direction of the straining part of the shear flow. Thus the change of the deformation parameter when

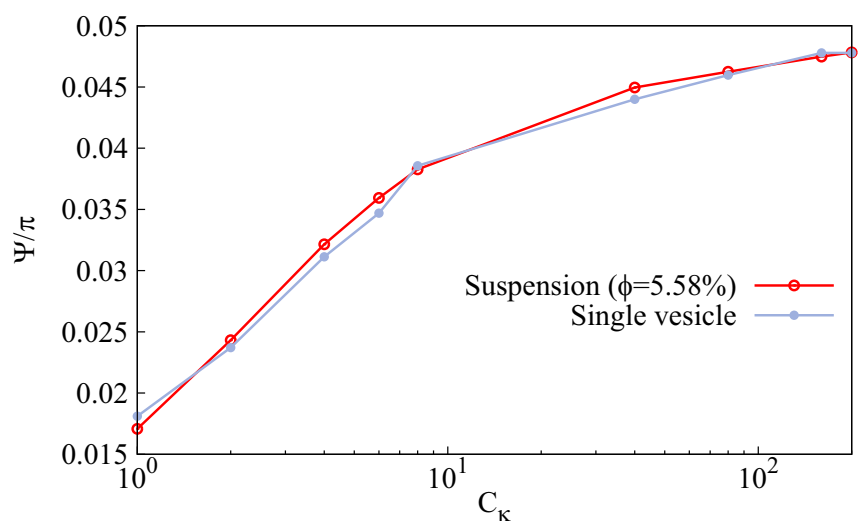

FIG. 8. Inclination angle of vesicles $(\phi=5.58 \%)$ as a function of $C_{\kappa}$ for a viscosity contrast $\lambda=10$. The angle increases with $C_{\kappa}$. 


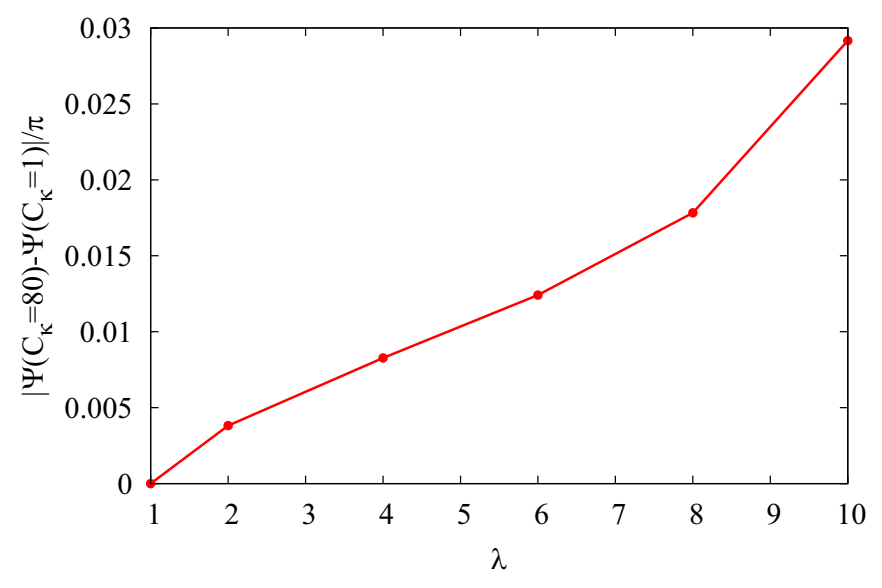

FIG. 9. Absolute difference of the inclination angle corresponding to two capillary numbers $\left(C_{\kappa}=1\right.$ and $\left.C_{\kappa}=80\right)$ as a function of the viscosity contrast $\lambda$.

increasing the shear rate has only a minor effect. In contrast, for high enough $\lambda$ (sufficiently close to the tumbling threshold) the inclination angle is small and thus is quite sensitive to variations of the shape of the vesicle which occur as a function of the capillary number. It follows that an increase of the shear rate, even if it induces a small reduction of the Taylor index, will cause a significant increase of the inclination angle. This tendency continues to operate until the angle has reached a value not too far from the maximum elongation direction of the straining component of the shear flow.

In summary, when $\lambda$ is rather small (far below the TT-TB transition), the main effect of the shear rate is the decrease of the Taylor deformation index (leading to shear thinning). When $\lambda$ is close to the value leading to the TT-TB transition, the effect of the Taylor deformation index is minor, and the main effect arises from an increase of the TT angle.

In order to investigate the evolution of the angle of TT $\Psi$ of vesicles of the suspension $(\phi=5.58 \%)$ as a function of capillary number $C_{\kappa}$, we plot the absolute difference of the angle for two values of the capillary number, $C_{\kappa}=1$ and $C_{\kappa}=$ 80 , for several viscosity contrasts (between $\lambda=1$ and $\lambda=10$ ).

As already introduced above, in the case of $\lambda=1$ the vesicles keep almost a constant TT angle upon increasing the capillary number $C_{\kappa}$, which leads to an almost zero absolute difference of the angle in this case, Fig. 9. By increasing viscosity contrast $\lambda$, the absolute difference of the TT angle between $C_{\kappa}=1$ and $C_{\kappa}=80$ is larger and larger as $\lambda$ increases, until it reaches a maximal explored value at $\lambda=10$, yielding the maximal absolute difference of the angle of about $\left|\Psi\left(C_{\kappa}=80\right)-\Psi\left(C_{\kappa}=1\right)\right| / \pi \simeq 0.03$. Beyond $\lambda=10$, for the degree of confinement $C_{n}=0.4$, the vesicles undergo a TB motion [19], a regime which is outside the scope of the present study.

Figures 10 and 11 show the streamlines for two extreme values of the capillary number. The first figure represents the streamlines of the full field, whereas the second one represents that of the induced field (that is, the full flow field from which we subtract the imposed shear flow). It is clearly seen that the streamlines are much more squeezed for the higher shear
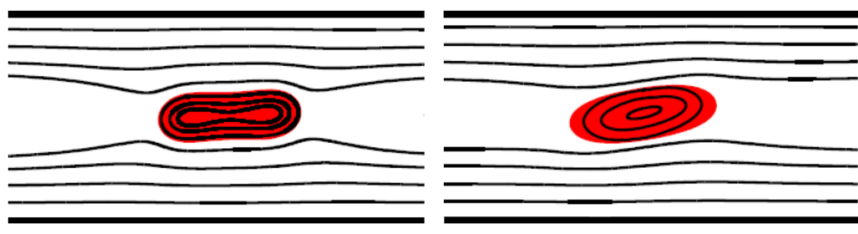

FIG. 10. Streamlines inside and outside a vesicle for the viscosity contrast $\lambda=10$, for two capillary numbers: $C_{\kappa}=1$ (left) and $C_{\kappa}=$ 200 (right). Here $(\lambda=10)$, the inclination angle increases with $C_{\kappa}$. The same set of values of the stream function is used for streamlines in the left and right panels.

rate, indicating a clear increase of hydrodynamical dissipation, leading to shear thickening.

For the sake of completeness, we have analyzed the evolution of the angle of a single vesicle and compared to that of a given vesicle in the suspension. This analysis is motivated by the determination of the contribution coming from a single entity and that arising from the interaction among vesicles. The results are reported in Fig. 8. It is seen that for a concentration of $5.58 \%$ the main effect comes from a single entity, and the effect of the interaction is quite minor.

Finally, it is interesting to note that the range of variation of the normalized effective viscosity for $\lambda=10$ (Fig. 6) remains below the range of the same quantity for $\lambda=1$ (Fig. 1), despite the fact that (i) the fluid is more viscous inside the vesicles in the first case, and (ii) the shear rate induces shear thickening. The first result is consistent with that initially reported in $[10,12]$ according to which the viscosity decreases for higher and higher viscosity contrast $\lambda$, provided the vesicle still remains in the TT regime. This effect is attributed to the fact that upon increasing $\lambda$ the inclination angle decreases, and thus the cross section against the fluid flow is reduced, leading to a lower viscosity. For $\lambda=10$, the vesicle inclination angle is close to zero, and the viscosity attains its minimal value. The shear rate causes the vesicle to straighten up (leading to shear thickening), but the inclination angle never reaches that obtained at $\lambda=1$. Thus the normalized effective viscosity for $\lambda=10$ remains always below that for $\lambda=1$.

It is noteworthy that the shear thinning and shear thickening effects are exhibited by a single vesicle. Figure 12 shows the results for a single vesicle for two values of reduced area: $v=0.7$ and $v=0.9$. In both cases we find that the suspension follows the same behavior as for the concentrations shown above. For $\lambda=1$ the suspension exhibits a shear thinning behavior and for $\lambda=10$ the suspension exhibits a shear thickening behavior. It is interesting also to note that for both

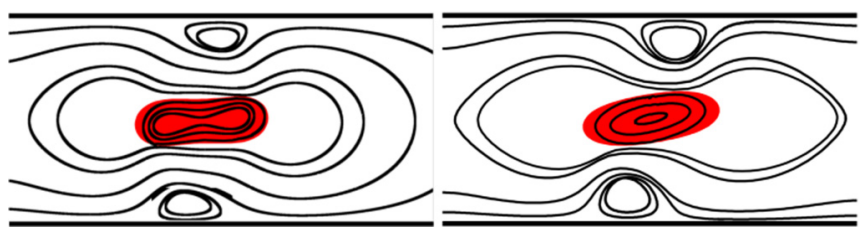

FIG. 11. Streamlines associated with the induced flow inside and outside a vesicle for $\lambda=10$, and for two capillary numbers: $C_{\kappa}=1$ (left) and $C_{\kappa}=200$ (right). The same set of values of the stream function is used for streamlines in the left and right panels. 

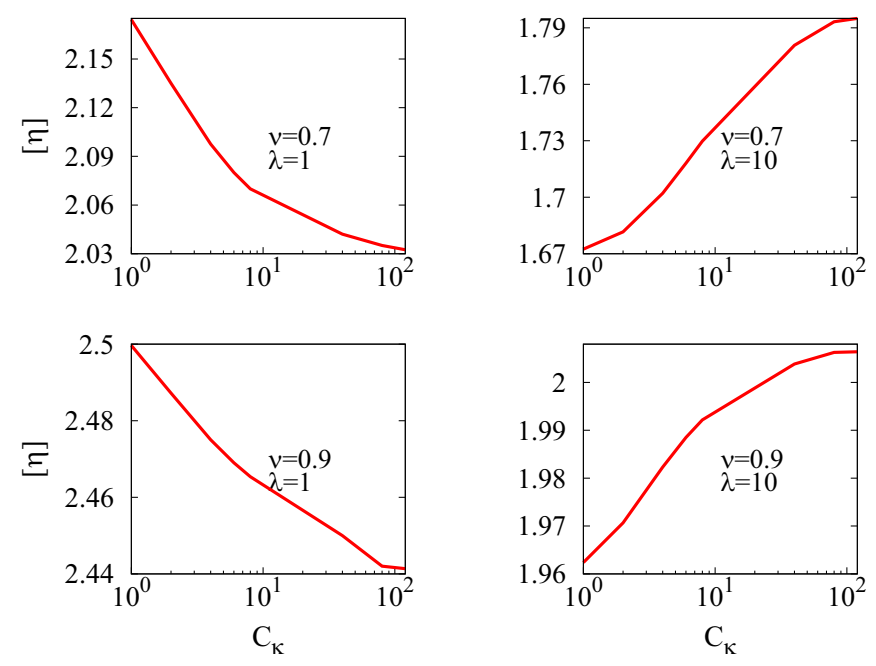

FIG. 12. The normalized effective viscosity $[\eta]$ as a function of capillary number $C_{\kappa}$ for a single vesicle for two values of reduced area: $v=0.7$ and $v=0.9$. For $\lambda=1(\lambda=10)$ the suspension exhibits a shear thinning (shear thickening) behavior regardless of the reduced area $v$.

cases the Taylor deformation index decreases with $C_{\kappa}$ and the inclination angle of the vesicle amply increases with $C_{\kappa}$ for $\lambda=10$, whereas the increase is minor for $\lambda=1$ (see Fig. 13). That this qualitative rheological behavior can be captured by a single vesicle model does not mean that the vesicle-vesicle interaction plays a minor role. Indeed, for a fixed shear rate, the normalized viscosity is a strongly nonlinear function of concentration, as reported in [27,37].

\section{Shear thinning-shear thickening crossover}

We have seen above that depending on $\lambda$ the same suspension shows either a shear thinning (low $\lambda$ ) or shear thickening (large $\lambda$ ). The question naturally arises of whether there is a critical $\lambda$ at which the suspension switches from one behavior to the other. For that purpose, we have analyzed the behavior of the normalized effective viscosity $[\eta]$ as a function of reduced shear rate $\left(C_{\kappa}\right)$ for several viscosity contrasts $\lambda$. The results are reported in Fig. 14. We have seen before that for $\lambda=1$, the suspension shows shear thinning. The results in Fig. 14 show that doubling the viscosity contrast already shows the birth of a weak shear thickening behavior, after a first interval
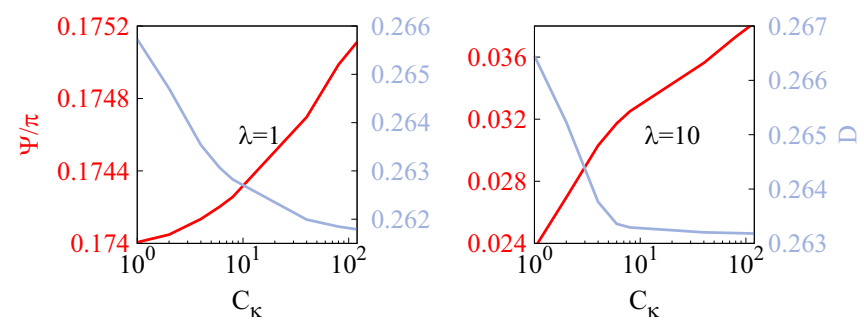

FIG. 13. The evolution of the Taylor deformation index $D$ and the inclination angle of the vesicle $\Psi$ as a function of capillary number $C_{\kappa}$ for reduced area $v=0.9$, for two values of viscosity contrasts: $\lambda=1$ and $\lambda=10$.
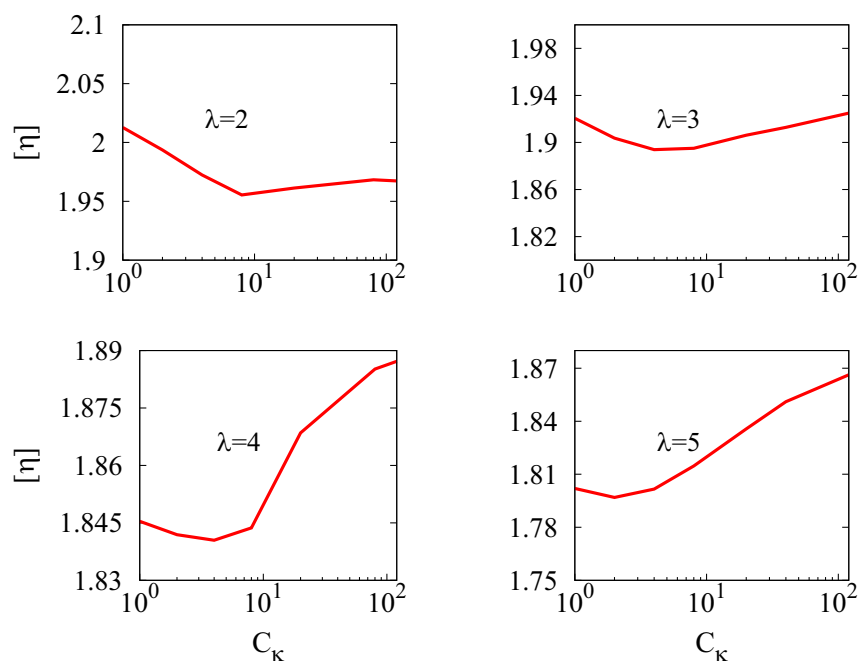

FIG. 14. Shear thinning to shear thickening crossover for a suspension with concentration $\phi=5.58 \%$.

of shear thinning. Increasing progressively $\lambda$ favors more and more the shear thickening behavior at the expense of the shear thinning one. For high enough $\lambda$ the shear thickening regime fully dominates.

As we have seen before there are two antagonistic effects: the decrease of the Taylor deformation index and straightening of the vesicles which favors shear thinning, and the increase of the TT angle which favors shear thickening. For low enough $\lambda$, say $\lambda=2$, one sees that a decrease of the Taylor deformation index $D$ with shear rate dominates over the increase of the TT angle (Fig. 15), whereas at larger $\lambda$ (say $\lambda=5$ and beyond) the opposite happens (Fig. 15). This analysis shows that the prevalence of shear thinning or shear thickening is quite subtle and requires an investigation of the behavior of the suspended entities under close scrutiny.

It can be generally expected that the effective viscosity of a suspension is affected by a multitude of factors, each acting in its own way. Since all of these factors vary upon changes of the shear rate, it is impossible to isolate their
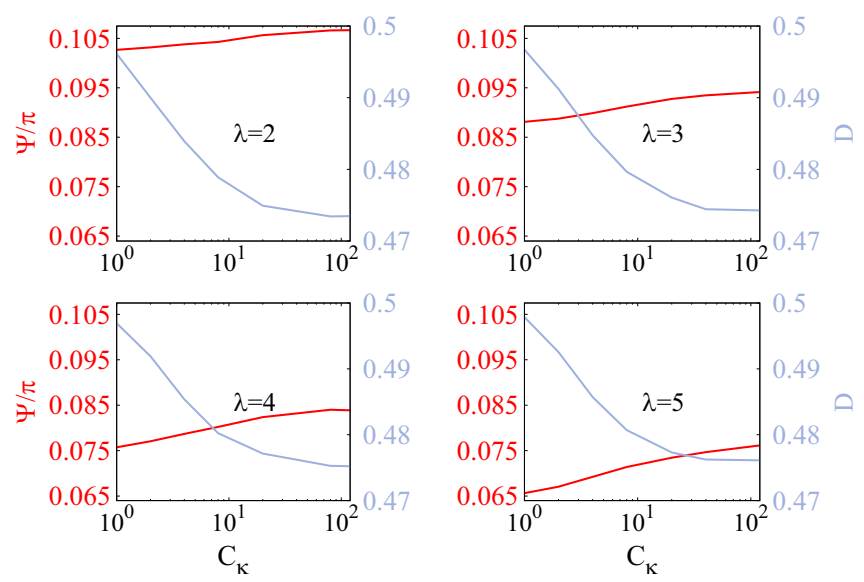

FIG. 15. The evolution of the Taylor deformation index $D$ and the inclination angle $\Psi$ of the vesicle as a function of capillary number $C_{\kappa}$ for different values of the viscosity contrast $\lambda$. 
effects independently. We cannot therefore provide a robust criterion which discriminates between shear thinning and shear thickening. For example, it has been observed in [42] for 2D vesicles at low $C_{\kappa}$ and in [43] for 3D vesicles that the TT angle decreases with increasing $C_{\kappa}$ for fixed $\lambda$. The range of $C_{\kappa}$ explored in [42] (a 2D study which is directly to be confronted to the present one) corresponds to the small capillary number regime $\left(C_{\kappa}=0.075-0.75\right)$. We have confirmed these results, which show an opposite behavior to that of the present results, which focused on higher $C_{\kappa}\left(C_{\kappa}=1-200\right)$. In other words, for a very small $C_{\kappa}$ the TT angle decreases when $C_{\kappa}$ is increased, while the opposite behavior is found here. This suggests that the full picture of vesicle dynamics and rheology is quite complex and that while the variations of the TT angle and of the Taylor deformation parameter can give useful insight into the rheological behavior of the suspension, there might be cases in which other factors play an important role.

Finally, it is interesting to note that the vesicle suspension can have a normalized effective viscosity $[\eta]$ which is significantly smaller than the Einstein one (for a dilute suspension of rigid disks, where $[\eta]=2$ ). We have seen here that for $\lambda>1$ we can achieve smaller viscosities as compared to the case with $\lambda=1$. Indeed, in Fig. 6 the minimal viscosity is of about 1.65, whereas for $\lambda=1$ (Fig. 1) it is equal to 2. As reported in [27] (where only $\lambda=1$ was treated), a minimal [ $\eta]$ can be achieved at a special concentration. It is expected that if we scan different concentrations in the case of $\lambda=10$, an even smaller value than 1.65 of $[\eta]$ can be achieved. Preliminary results show that indeed this minimal value can be significantly lowered. We hope to report along these lines, and more generally on the problem of monitoring a suspension that yields the smallest viscosity.

\section{CONCLUSION}

To sum up, we have presented numerical simulations of the rheological behavior of a confined suspension of vesicles under shear flow as a function of capillary number $C_{\kappa}$. Our simulations are based on the boundary integral method (2D). Special Green's functions are used in order to take into account the no-slip boundary condition at the bounding walls. The suspension of vesicles can either show shear thinning behavior, which is related to the deformability of vesicles in flow, or shear thickening behavior, which is related to the increase of TT angle of vesicles. We have also seen that the rheological system undergoes a gradual transition from a shear thinning to a shear thickening behavior upon increasing the viscosity contrast. Several blood diseases (such as sickle cell disease and malaria) are accompanied by an increase of the cytoplasm viscosity. It would be interesting to investigate the shear-dependent rheology in confined suspensions for various RBC pathologies. Note that blood in unconfined geometry always shows a shear thinning behavior, partly due to the dissociation $\mathrm{RBC}$ rouleaux and partly due to the RBC deformation. The study of rheology reported here has shown that the situation is more complex with confined suspensions than with the unbounded case. This analysis introduces a paradigm that opens a way for a refined analysis of confined rheology. Finally, we would like to draw attention to the fact that the normalized effective viscosity undergoes here only moderate changes with shear rate (of about $10 \%-15 \%$ ), whereas the normalized normal stress difference can vary by a factor of up to two (see Fig. 6). Here we have focused our study on the case of a $2 \mathrm{D}$ suspension. A preliminary study presented in [27] has shown that the same type of order of the suspending entities takes place also in 3D. It is natural to expect a similar behavior regarding the shear thinning and shear thickening behavior to that in 2D. In addition, the reduced volume of a RBC is lower than that analyzed here (the reduced volume for a RBC is about 0.6), and the deformation amplitudes, due to the dimensionality, may be significantly stronger than those reported here. This quantitative difference may trigger ampler manifestation of the rheological signatures. We hope to investigate this matter in the future.

\section{ACKNOWLEDGMENTS}

We thank CNES (Centre National d'Etudes Spatiale), ESA (European Space Agency), the French-German university program "Living Fluids" (Grant No. CFDA-Q1-14), and CNRST-Morocco (Grant Ref. k 1/045) for financial support. C.M. thanks CNRST (project FINCOM). The simulations were performed on the Cactus cluster of the CIMENT infrastructure, which is supported by the Rhône-Alpes region (Grant No. CPER07_13 CIRA).
[1] R. Lipowsky and E. Sackmann, Structure and Dynamics of Membranes: I. From Cells to Vesicles/II. Generic and Specific Interactions (Elsevier, North Holland, 1995), Vol. 1.

[2] H. Schmid-Schönbein, R. Wells, and J. Goldstone, Circ. Res. 25, 131 (1969).

[3] L. Dintenfass, Nature (London) 219, 956 (1968).

[4] H. H. Lipowsky, Microcirculation 12, 5 (2005).

[5] S. Chien, Science 168, 977 (1970).

[6] S. Chien, S. Usami, R. J. Dellenback, M. I. Gregersen, L. B. Nanninga, and M. M. Guest, Science 157, 829 (1967).

[7] S. Chien, S. Usami, R. J. Dellenback, and M. I. Gregersen, Science 157, 827 (1967).
[8] A. M. Robertson, A. Sequeira, and R. G. Owens, in Cardiovascular Mathematics (Springer, Italia, Milano, 2009), pp. 211-241.

[9] R. G. Larson, The Structure and Rheology of Complex Fluids (Oxford University Press, New York, 1999).

[10] C. Misbah, Phys. Rev. Lett. 96, 028104 (2006).

[11] P. M. Vlahovska and R. S. Gracia, Phys. Rev. E 75, 016313 (2007).

[12] G. Danker and C. Misbah, Phys. Rev. Lett. 98, 088104 (2007).

[13] G. Ghigliotti, H. Selmi, B. Kaoui, G. Biros, and C. Misbah, ESAIM: Proc. 28, 212 (2009).

[14] G. Ghigliotti, T. Biben, and C. Misbah, J. Fluid Mech. 653, 489 (2010).

[15] S. K. Doddi and P. Bagchi, Phys. Rev. E 79, 046318 (2009). 
[16] P. Bagchi and R. M. Kalluri, Phys. Rev. E 80, 016307 (2009).

[17] P. Bagchi and R. M. Kalluri, Phys. Rev. E 81, 056320 (2010).

[18] A. Lamura and G. Gompper, Proc. IUTAM 16, 3 (2015).

[19] M. Thiébaud and C. Misbah, Phys. Rev. E 88, 062707 (2013).

[20] V. Kantsler and V. Steinberg, Phys. Rev. Lett. 95, 258101 (2005).

[21] V. Vitkova, M.-A. Mader, B. Polack, C. Misbah, and T. Podgorski, Biophys. J. 95, L33 (2008).

[22] R. Kalluri and P. Bagchi, in APS Meeting Abstracts, 2011, Vol. 1, p. 25010.

[23] D. Matsunaga, Y. Imai, T. Omori, T. Ishikawa, and T. Yamaguchi, J. Biomech. Sci. Eng. 9, 14 (2014).

[24] D. Reasor, J. Clausen, and C. Aidun, J. Fluid Mech. 726, 497 (2013).

[25] D. A. Fedosov, H. Noguchi, and G. Gompper, Biomech. Model. Mechan. 13, 239 (2014).

[26] D. Matsunaga, Y. Imai, T. Yamaguchi, and T. Ishikawa, J. Fluid Mech. 786, 110 (2016).

[27] M. Thiébaud, Z. Shen, J. Harting, and C. Misbah, Phys. Rev. Lett. 112, 238304 (2014).

[28] B. Kaoui, R. J. Jonk, and J. Harting, Soft Matter 10, 4735 (2014).

[29] A. Lamura and G. Gompper, Europhys. Lett. 102, 28004 (2013).

[30] B. Kaoui, J. Harting, and C. Misbah, Phys. Rev. E 83, 066319 (2011).
[31] B. Kaoui, G. H. Ristow, I. Cantat, C. Misbah, and W. Zimmermann, Phys. Rev. E 77, 021903 (2008).

[32] O.-Y.Zhong-Can and W. Helfrich, Phys. Rev. A 39, 5280 (1989).

[33] C. Pozrikidis, Boundary Integral and Singularity Methods for Linearized Viscous Flow (Cambridge University Press, Cambridge, 1992).

[34] G. Batchelor, J. Fluid Mech. 41, 545 (1970).

[35] A. M. Forsyth, J. Wan, P. D. Owrutsky, M. Abkarian, and H. A. Stone, Proc. Natl. Acad. Sci. USA 108, 10986 (2011).

[36] L. Lanotte, J. Mauer, S. Mendez, D. A. Fedosov, J.-M. Fromental, V. Claveria, F. Nicoud, G. Gompper, and M. Abkarian, Proc. Natl. Acad. Sci. USA 113, 13289 (2016).

[37] Z. Shen, A. Farutin, M. Thiébaud, and C. Misbah, Phys. Rev. Fluids 2, 103101 (2017).

[38] O. Aouane, A. Farutin, M. Thiébaud, A. Benyoussef, C. Wagner, and C. Misbah, Phys. Rev. Fluids 2, 063102 (2017).

[39] M. Kennedy, C. Pozrikidis, and R. Skalak, Comput. Fluids 23, 251 (1994).

[40] D. Barthes-Biesel and A. Acrivos, J. Fluid Mech. 61, 1 (1973).

[41] B. Kaoui, T. Krüger, and J. Harting, Soft Matter 8, 9246 (2012).

[42] J. Beaucourt, F. Rioual, T. Séon, T. Biben, and C. Misbah, Phys. Rev. E 69, 011906 (2004).

[43] B. Kaoui, A. Farutin, and C. Misbah, Phys. Rev. E 80, 061905 (2009). 